\title{
Recent advances in singular boundary method for ultra-thin structural problems
}

\author{
Y. Gu \& W. Chen \\ Department of Engineering Mechanics, Hohai University, China
}

\begin{abstract}
This study makes the first attempt to apply the singular boundary method (SBM), a meshless boundary collocation method, for the analysis of two-dimensional (2D) thin structural problems. The troublesome near-singular kernels, which are crucial in the applications of SBM to thin shapes, are dealt with efficiently by using a non-linear transformation technique. Promising SBM results with only a small number of elements are obtained for thin films and coatings with the thickness-to-length ratio is as small as 1E-9, which is sufficient for modeling most thin structures as used in smart materials and micro-electro-mechanical systems. The advantages, disadvantages and potential applications of the proposed method, as compared with the finite element (FEM) and boundary element (BEM) methods, are also discussed.

Keywords: singular boundary method, meshless boundary collocation method, fundamental solutions, non-linear coordinate transformation, thin-walled structures.
\end{abstract}

\section{Introduction}

The study of boundary value problems for thin-walled structures has received considerable attention in recent years. This interest is partly related to the extensive use of smart materials and micro-electro-mechanical systems (MEMS) in various engineering applications. Analysis of this kind of problem is, however, very difficult because the structures are usually made in the forms of ultra-thin films with the thickness-to-length ratios in the micro (1E-6) or nano (1E-9) scales.

The FEM has long been a dominant numerical technique in the simulation of many industrial problems. However, the aspect ratio issues associated with the 
FEM when applied to thin structures limit its application. To maintain element aspect ratio, a large number of elements must be discretized, and the procedure therefore requires much preprocessing and CPU time as the thickness decreases. As an alternative approach, the BEM has long been believed to avoid such drawbacks due to the boundary-only discretizations and its semi-analytical nature. It has been shown in $[1,2]$ that the BEM can deal with thin structures efficiently as long as the near-singular integrals existing in the BEM formulations are handled correctly. In the past two decades, numerous research works have been published on this subject in the BEM literature. However, most of these earlier methods are either inefficient or cannot provide accurate results when the ratio of the thickness is smaller than $1 \mathrm{E}-6$.

The singular boundary method (SBM) belongs to the family of meshless boundary collocation method [3-6] and can be viewed as one kind of modified method of fundamentals (MFS). This method fully inherits the merits of the MFS being truly meshless, integration-free, easy-to-program and in the meantime overcoming the bottleneck fictitious boundary issue associated with the traditional MFS. Prior to this study, this method has since been successfully applied to a variety of physical problems, such as two- and three-dimensional potential problems $[7,8]$, infinite domain problems [9], and elasticity problems [10].

This paper is an extension of our previous work [11] where a non-linear transformation was proposed and applied to treat boundary layered effect occurring in 2D potential problems. Herein, the developed algorithm is extended to the numerical analysis of 2D thin structural problems. For the text problems studied, very promising results are obtained when the thickness to length ratio is in the orders of $1 \mathrm{E}-1$ to $1 \mathrm{E}-9$, which is sufficient for modeling most thin structures in nano-scales. A brief outline of the rest of this paper is as follows. The SBM formulation and its implementation are presented in Section 2. Section 3 introduces a nonlinear transformation, based on sinh function, to remove or damp out the near singular properties of fundamental solutions. Followed in Section 4, the accuracy and efficiency of the proposed method are tested to two benchmark 2D thin structural problems, in which the proposed method is compared with the FEM and BEM. Finally, the conclusions and remarks are provided in Section 5.

\section{The SBM formulation for 2D potential problems}

Without a loss of generality, we introduce the SBM formulation with Laplace equation governing potential problems as follows:

$$
\nabla^{2} u(\boldsymbol{x})=0, \boldsymbol{x} \in \Omega,
$$

subject to the following boundary conditions

$$
\begin{gathered}
u(\boldsymbol{x})=\bar{u}(\boldsymbol{x}), \quad \boldsymbol{x} \in \Gamma_{\mathrm{D}} \text { (Dirichlet boundary condition), } \\
q(\boldsymbol{x})=\frac{\partial u}{\partial \boldsymbol{n}}(\boldsymbol{x})=\bar{q}(\boldsymbol{x}), \quad \boldsymbol{x} \in \Gamma_{\mathrm{N}}(\text { Neumann boundary condition }),
\end{gathered}
$$


where $u$ is the potential field, $\Gamma_{\mathrm{D}}$ and $\Gamma_{\mathrm{N}}$ construct the whole boundary of the domain $\Omega, \boldsymbol{n}$ denotes the outward normal, and the barred quantities indicate the given values on the boundary.

The MFS approximates the solution $u(\boldsymbol{x})$ and $q(\boldsymbol{x})$ by a linear combination of fundamental solutions with respect to different source points $\boldsymbol{s}^{j}$ :

$$
\begin{gathered}
u\left(\boldsymbol{x}^{i}\right)=\sum_{j=1}^{N} \alpha^{j} A\left(\boldsymbol{x}^{i}, \boldsymbol{s}^{j}\right), \\
q\left(\boldsymbol{x}^{i}\right)=\frac{\partial u\left(\boldsymbol{x}^{i}\right)}{\partial \boldsymbol{n}_{\boldsymbol{x}^{i}}}=\sum_{j=1}^{N} \alpha^{j} \frac{\partial A\left(\boldsymbol{x}^{i}, \boldsymbol{s}^{j}\right)}{\partial \boldsymbol{n}_{\boldsymbol{x}^{i}}},
\end{gathered}
$$

where $\boldsymbol{x}^{i}$ is the $i^{\text {th }}$ collocation point and $\boldsymbol{s}^{j}$ is the $j^{\text {th }}$ source point placed on a fictitious boundary outside $\Omega, \alpha^{j}$ denotes the $j^{\text {th }}$ unknown coefficient of the distributed source at $\boldsymbol{s}^{j}, N$ represents the numbers of source points, and

$$
A\left(\boldsymbol{x}^{i}, \boldsymbol{s}^{j}\right)=-\frac{1}{2 \pi} \ln \left\|\boldsymbol{x}^{i}-\boldsymbol{s}^{j}\right\|_{2},
$$

is the fundamental solution associated with 2D Laplace equation.

Similar to the MFS, the SBM also uses the fundamental solution as the basis kernel function of its approximation. In stark contrast to the MFS, the collocation and source points of the SBM are coincident and are placed on the real boundary without using a fictitious boundary. The basic idea of this method is to introduce a concept of the origin intensity factors to isolate the singularity of the fundamental solutions, so that the source points can be placed on the real boundary directly. With this idea in mind we represent the SBM interpolation as

$$
\begin{gathered}
u\left(\boldsymbol{x}^{i}\right)=\sum_{j=1, i \neq j}^{N} \alpha^{j} A\left(\boldsymbol{x}^{i}, \boldsymbol{s}^{j}\right)+\alpha^{i} u_{i i}, \\
q\left(\boldsymbol{x}^{i}\right)=\sum_{j=1, i \neq j}^{N} \alpha^{j} \frac{\partial A\left(\boldsymbol{x}^{i}, \boldsymbol{s}^{j}\right)}{\partial \boldsymbol{n}_{\boldsymbol{x}^{i}}}+\alpha^{i} q_{i i} .
\end{gathered}
$$

where $u_{i i}$ and $q_{i i}$ are defined as the origin intensity factors, i.e., the diagonal elements of the SBM interpolation matrix. Observe that for $\boldsymbol{x}=\boldsymbol{s}, u_{i i}$ and $q_{i i}$ are singular. In the SBM method, the weakly singular kernels are determined by the integration of the fundamental solution on line segments, and the use of the desingularization of subtracting and adding-back technique for the calculation of diagonal coefficients from the derivatives of the fundamental solutions. The main results for 2D potential problems are summarized hereafter. First, the origin intensity factors on Dirichlet boundary conditions are given by

$$
u_{i i}=\frac{1}{l_{i}} \int_{\Gamma_{s}} A\left(x^{i}, s\right) d \Gamma_{s}=-\frac{1}{2 \pi l_{i}} \int_{\Gamma_{s}} \ln \left\|x^{i}-s\right\|_{2} d \Gamma_{s} \approx \frac{1}{2 \pi}\left(\ln \frac{2}{l_{i}}+1\right),
$$

where $l_{j}$ denotes the half distance between the source nodes $\boldsymbol{s}^{j-1}$ and $\boldsymbol{s}^{j+1}$. 
The final results for Neumann boundary equations, after some analytical manipulation, is

$$
q\left(\boldsymbol{x}^{i}\right)=\sum_{j=1, i \neq j}^{N} \alpha^{j} \frac{\partial A\left(\boldsymbol{x}^{i}, \boldsymbol{s}^{j}\right)}{\partial \boldsymbol{n}_{\boldsymbol{x}^{i}}}-\frac{\alpha^{i}}{l_{i}} \sum_{j=1, i \neq j}^{N} l_{j} \frac{\partial A\left(\boldsymbol{x}^{i}, \boldsymbol{s}^{j}\right)}{\partial \boldsymbol{n}_{\boldsymbol{s}^{j}}},
$$

where,

$$
q_{i i}=-\frac{1}{l_{i}} \sum_{j=1, i \neq j}^{N} l_{j} \frac{\partial A\left(\boldsymbol{x}^{i}, \boldsymbol{s}^{j}\right)}{\partial \boldsymbol{n}_{\boldsymbol{s}^{j}}} .
$$

We refer readers to Refs. $[11,12]$ for detailed derivations of the above eqns (9) and (11).

Using the procedures described above, the origin intensity factors on both the Neumann and Dirichlet boundary equations have been extracted out. Once all the boundary unknowns are solved, the potential and its derivative at any point inside the domain can be evaluated using the following equations

$$
\begin{gathered}
u(\boldsymbol{y})=\sum_{j=1}^{N} \alpha^{j} A\left(\boldsymbol{y}, \boldsymbol{x}^{j}\right), \\
\nabla_{\boldsymbol{y}} u(\boldsymbol{y})=\sum_{j=1}^{N} \alpha^{j} \nabla_{\boldsymbol{y}} A\left(\boldsymbol{y}, \boldsymbol{x}^{j}\right),
\end{gathered}
$$

where $\boldsymbol{x}^{j} \in \Gamma, \boldsymbol{y}$ is a field point located inside the domain, and $\nabla_{\boldsymbol{y}}$ stands for the partial derivatives with respect to $\boldsymbol{y}$. Employing indicial notation for the coordinates of point $\boldsymbol{y}$, i.e. $y_{1}, y_{2}, \nabla_{\boldsymbol{y}}$ can be written as $\partial / \partial y_{1}$ or $\partial / \partial y_{2}$.

\section{Treatment of nearly singular terms}

When the calculation point $\boldsymbol{y}$ is far away from the boundary, a straightforward application of the SBM-expansion (eqns (12) and (13)) suffices to obtain accurate numerical results. However, when the calculation point is close to, but not on, the boundary, the kernel functions will present various orders of near singularities. In such cases, the kernels remains finite at all points. However, instead of remaining flat, the kernels develop a sharp peak as the collocation point moves closer to the boundary, thus rendering accurate evaluation of the kernels difficult. This is very similar to the so-called boundary layer effect associated with the BEM-based methods.

The near-singular kernels occur always in two situations: first one is known as boundary layer effect problems when the physical quantities at the interior points very close to boundary are calculated. The other one is named as thin structural problems when the thickness of the considered domain is small. In our previous work [11], a non-linear transformation for evaluating near-singular kernels was proposed and the troublesome boundary layer effect related to SBM has been successfully remedied. Based on the method developed in [11], the 
SBM formulation and computer code for thin structural problems are developed in this paper.

For a thin structure, some boundary nodes on one side of the surface will be very close to the nodes on the opposite side, as shown in Figure 1. Thus, the distance $r$ between some boundary nodes will approach zero. This gives rise to near-singular kernels in boundary equations (7) and (8), which are to be regularized together with usual singular kernels. Moreover, almost all the interior points of a thin structure are very close to the boundary, leading equations (12) and (13) nearly singular. Therefore, in order to apply the SBM to thin structural problems, one has to deal with near-singular kernels arising in both equations (7) and (8) for boundary unknowns and equations (12) and (13) for interior

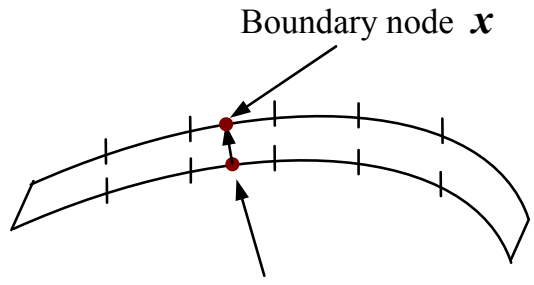

Boundary node $\boldsymbol{x}^{o}$

Figure 1: $\quad$ Sketch of a 2D thin structure in which points $\boldsymbol{x}^{\circ}$ and $\boldsymbol{x}$ represent boundary points on the adjoining boundaries.

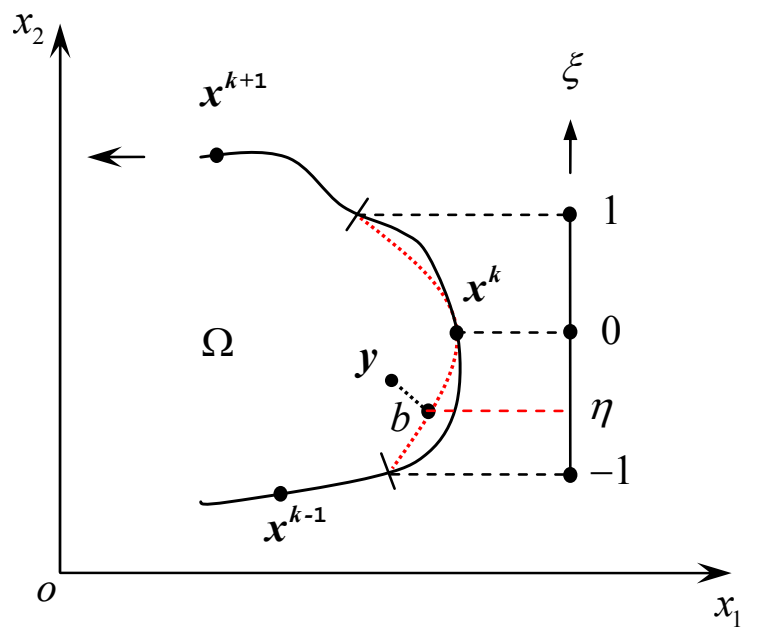

Figure 2: Near-boundary point $\boldsymbol{y}$. 
quantities, different to the boundary layer effect where only equations (12) and (13) need to be considered.

As shown in Figure 2, we suppose the field point $\boldsymbol{y}$ is very close to a portion of the boundary containing the point $\boldsymbol{x}^{k}$, then we can rewrite eqns (12) and (13) as

$$
\begin{gathered}
u(\boldsymbol{y})=\sum_{j=1, j \neq k}^{N} \alpha^{j} A\left(\boldsymbol{y}, \boldsymbol{x}^{j}\right)+\alpha^{k} u_{k}, \\
\nabla_{\boldsymbol{y}} u(\boldsymbol{y})=\sum_{j=1, j \neq k}^{N} \alpha^{j} \nabla_{\boldsymbol{y}} A\left(\boldsymbol{y}, \boldsymbol{x}^{j}\right)+\alpha^{k} q_{k},
\end{gathered}
$$

where $u_{k}$ and $q_{k}$ are defined as the nearly singular factors which should be regularized by using some special treatment.

In this study, the nearly singular factors $u_{k}$ and $q_{k}$ are directly calculated as an average value of the fundamental solution over a portion of boundary. This can be formed as

$$
u_{k}=\frac{1}{l_{k}} \int_{\Gamma_{k}} A(\boldsymbol{y}, \boldsymbol{x}) d \Gamma_{k}(\boldsymbol{x}), q_{k}=\frac{1}{l_{k}} \int_{\Gamma_{k}} \nabla_{\boldsymbol{y}} A(\boldsymbol{y}, \boldsymbol{x}) d \Gamma_{k}(\boldsymbol{x}),
$$

where $l_{k}$ is half distance between the source nodes $\boldsymbol{x}_{\boldsymbol{k}-1}$ and $\boldsymbol{x}_{\boldsymbol{k}+1}$, see Figure 2 .

The above integral eqn (16) are numerically calculated by approximating the geometry segment $\Gamma_{k}(\boldsymbol{x})$ using interpolation polynomials and transforming the integrals so that they are mapped onto the interval $[-1,1]$ in terms of some intrinsic coordinate $\xi$. If quadratic boundary element is employed, the distance $r$ between the field point and the element becomes [13]

$$
r=\sqrt{(\xi-\eta)^{2} g(\xi)+b^{2}},
$$

where $\eta \in[-1,1]$ represents the position of the projection of the field point onto the element (see Figure 2), $b$ denotes the shortest distance from the field point to the boundary, $g(\xi) \geq 0$ is a low-order polynomial. We refer interested readers to Ref. [13] for further details. Using the procedure described above, we can rewrite eqn (16) as follows

$$
\begin{gathered}
u_{k}=-\frac{1}{4 \pi l_{k}} \int_{-1}^{1} J(\xi) \ln \left[(\xi-\eta)^{2} g(\xi)+b^{2}\right] d \xi, \\
q_{k}=-\frac{1}{2 \pi l_{k}} \int_{-1}^{1} \frac{f(\xi) J(\xi)}{(\xi-\eta)^{2} g(\xi)+b^{2}} d \xi,
\end{gathered}
$$

where $J(\xi)$ represents the Jacobian of the transformation, $f(\bullet)$ is a low-order polynomial which arises from taking the derivative of the boundary element kernel. It is obvious that the above integrals would present various orders of near singularity if $b$ is very small. To achieve high accuracy, the key procedure is to 
find an algorithm to accurately calculate these nearly singular integrals for a small value of $b$.

As mentioned above, the nearly singular integrals under consideration contain an argument of the form $(\xi-\eta)^{2} g(\xi)+b^{2}$. For evaluating an integral of this type, Gu et al. [11] suggest a change of integration variable using a sinh function [14]

$$
\xi=\eta+b \sinh \left(k_{1} t+k_{2}\right),
$$

where $k_{1}$ and $k_{2}$ are chosen such that the transformation maps [-1, 1] onto $[-1,1]$ so that the Gaussian quadrature can be applied in a straightforward fashion to the transformed integral. The Jacobian of transformation (20) is given by

$$
\frac{d \xi}{d t}=b k_{1} \cosh \left(k_{1} t-k_{2}\right) .
$$

Substituting the transformation (20) into the integrals (18) and (19) yields

$$
\begin{gathered}
u_{k}=-\frac{b k_{1}}{4 \pi l_{k}} \int_{-1}^{1} J(t) \cosh \left(k_{1} t-k_{2}\right) \ln \left\{b^{2}\left[\sinh ^{2}\left(k_{1} t-k_{2}\right) g(t)+1\right]\right\} d t \\
q_{k}=-\frac{k_{1}}{2 \pi l_{k} b} \int_{-1}^{1} \frac{f(t) J(t) \cosh \left(k_{1} t-k_{2}\right)}{\sinh ^{2}\left(k_{1} t-k_{2}\right) g(t)+1} d t
\end{gathered}
$$

In the above eqns (22) and (23), the function $\sinh ^{2}\left(k_{1} t-k_{2}\right) g(t)+1$ is always greater than 1 since $g(t)$ is a non-negative function as mentioned above. Thus, the integrands are fully regularized and can now be computed straightforward via the standard Gaussian quadrature, even if the value of $b$, distance between boundary source and inner collocation points, is very small.

It should be noted that the nearly singular kernels are calculated by integrating the fundamental solutions over the analyzed surface. However, such numerical integrations only increase negligible computing costs compared with the total costs since the integration is only used for nearly singular kernels. In fact, we calculate all SBM interpolation matrix elements directly in the strongform fashion as in the MFS. We only calculate these nearly singular terms by using numerical integration, and therefore, the developed SBM for thin structural problems remains a strong form approach.

\section{Numerical results and discussions}

We consider the heat diffusion in a thin structure with amoeba-like shapes, as shown in Figure 3. In this example, the distance $(b)$ between the outer and inner boundaries is defined as the thickness-to-length ratio which varies in the range of $10^{-1} \leq b \leq 10^{-10}$. The specified boundary conditions are temperatures on outer boundary and normal fluxes on the rest of the boundary. The exact solution is available as

$$
u(\boldsymbol{x})=\sin x_{1} \cosh x_{2}+x_{1}^{2}-x_{2}^{2}+x_{1} x_{2} .
$$




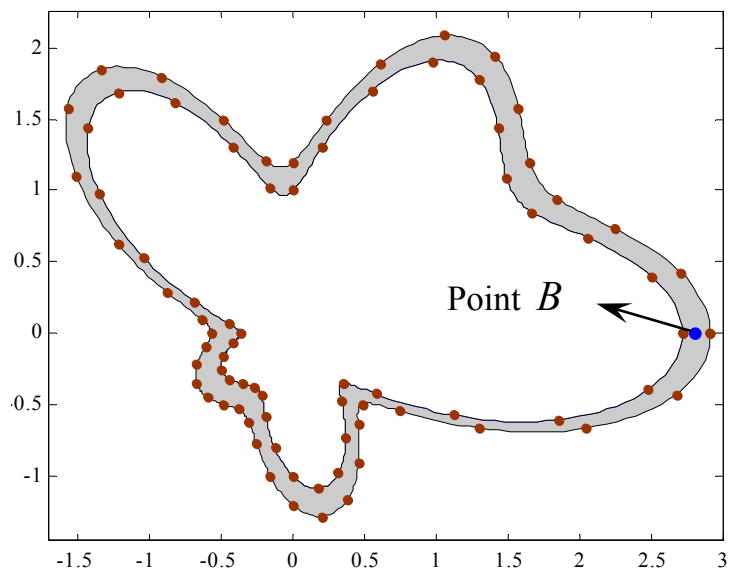

Figure 3: A thin structure with amoeba-like shapes.

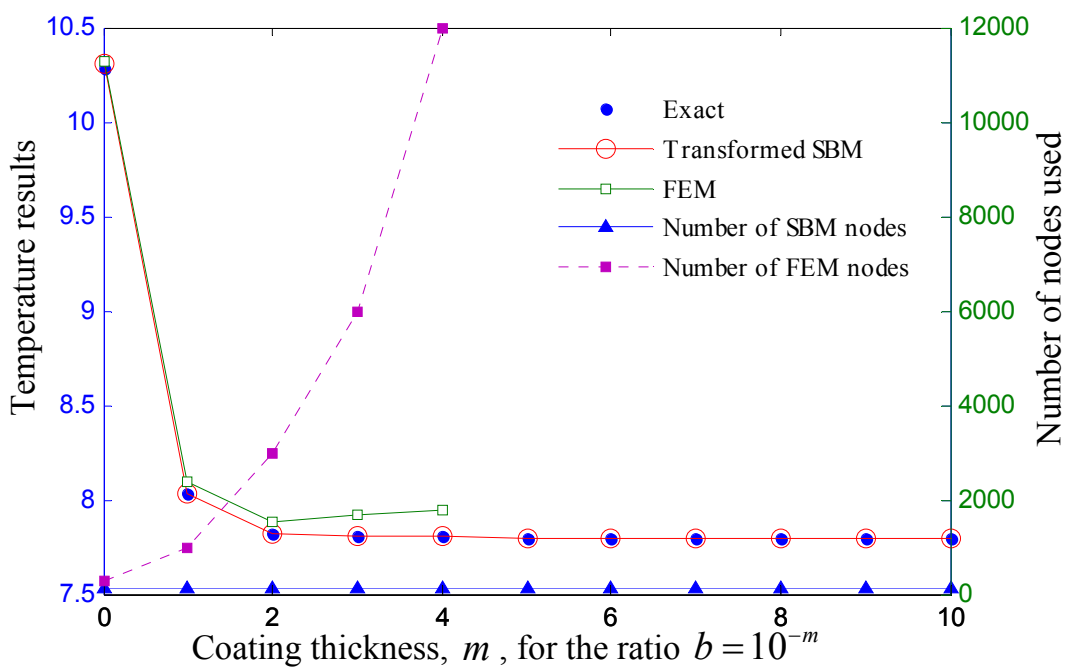

Figure 4: Temperature results and numbers of nodes required for different thickness-to-length ratio (Note that the smallest thickness solved is: $b=10^{-10}$ for SBM and $b=10^{-4}$ for FEM).

For the SBM model, $N=100$ evenly distributed boundary nodes are chosen on the boundary, regardless of the thickness of the structure. The FEM discretization varies according to the structure thickness and, generally, as the thickness decreases, more finite elements are required in order to maintain reasonable element aspect ratio. Figure 4 shows the temperature results at the 
point $A$, and also the number of nodes needed to achieve solution for both SBM and FEM. We see that even for thickness-to-length ratio $b$ reaches $1 \mathrm{E}-9$, the SBM results are still very good, almost reproducing the exact values. Again, the number of SBM nodes does not change across the entire range of thickness-tolength ratio. The solution time and memory requirements are therefore quite modest for the SBM procedure. The FEM solution, however, demonstrates a very different behavior. While the cases with $b \geq 0.1$ are easily solved with the FEM, with a fairly small number of elements, the solution requires significantly more effort when the value of $b$ less than 0.1 . Indeed, for $b<10^{-4}$, the FEM solution becomes infeasible due to memory limitations. Generally speaking, as has already illustrated in Refs. [15-18] or other studies in FEM or BEM literature, the number of FEM elements increases rapidly for thin structures due to aspect ratio limitations, and consequently, the FEM eventually becomes infeasible due to memory constraints. On the other hand, the proposed SBM does not require a refined mesh and can continue to provide accurate results for $b=10^{-10}$ without any difficulty. Similar results have also been obtained for the flux results, as illustrated in Table 1. Hence we can conclude that, in comparison with existing methods for solving thin structural problems, the SBM could be considered a competitive alternative.

Table 1: $\quad$ Results of fluxes $\partial u / \partial x_{1}$ at the point $A$ using SBM and BEM.

\begin{tabular}{|c|c|c|c|c|}
\hline$b$ & Exact & Untransformed SBM & Transformed SBM & $\begin{array}{c}\text { BEM } \\
\text { Ref. [13] }\end{array}$ \\
\hline $1 \mathrm{E}-1$ & 4.6054 & 5.8743 & 4.6145 & 4.6214 \\
\hline $1 \mathrm{E}-2$ & 4.5327 & $\times$ & 4.5369 & 4.5769 \\
\hline $1 \mathrm{E}-3$ & 4.5256 & $\times$ & 4.5259 & 4.5821 \\
\hline $1 \mathrm{E}-4$ & 4.5249 & $\times$ & 4.5247 & 4.5953 \\
\hline $1 \mathrm{E}-5$ & 4.5248 & $\times$ & 4.5246 & 4.5997 \\
\hline $1 \mathrm{E}-6$ & 4.5248 & $\times$ & 4.5246 & 4.6001 \\
\hline $1 \mathrm{E}-7$ & 4.5248 & $\times$ & 4.5245 & 4.6012 \\
\hline $1 \mathrm{E}-8$ & 4.5248 & $\times$ & 4.5246 & 4.6098 \\
\hline $1 \mathrm{E}-9$ & 4.5248 & $\times$ & 4.5249 & 4.6114 \\
\hline $1 \mathrm{E}-10$ & 4.5248 & $\times$ & 4.5246 & 4.6953 \\
\hline
\end{tabular}

\section{Conclusions and remarks}

This paper investigates the applicability of the SBM for the analysis of 2D thin structures in micro- and nano-scales. It is shown that the SBM, with proper treatment of near-singular kernels, is very accurate and efficient in modeling 2D thin structural problems. For all the test problems studied, the SBM provides a computationally-efficient solution for both temperature and flux fields, and in general, outperforms the BEM and FEM in terms of overall accuracy and 
efficiency. Compared with BEM and FEM, the developed SBM has the following attractive features:

(i) Using only boundary discretization instead of domain discretization, the SBM does not suffer from thickness or aspect ratio issues associated with FEMbased methods. Therefore, the number of SBM nodes can be held constant as described in example, without any loss in solution accuracy as the thickness decreases.

(ii) All SBM interpolation matrix elements are directly calculated in the strong-form fashion and only near-singular terms are determined by numerical integration. Therefore, the develop method remains a strong form approach and is computationally far more efficient, easier-to-program and mathematically simpler as compared with the BEM.

All these features of the SBM approach make it very attractive to the modeling and analysis of thin structural problems. Applications of the proposed method to thin structural problems can be found in many areas such as thinlayered coating systems, turbine blades, rudders and various containers, where other computational modes, such as the FEM, become inefficient or fail. Some work along this line is already underway and will be reported in a subsequent paper.

\section{Acknowledgements}

The work described in this paper was supported by the National Basic Research Program of China (973 Project No. 2010CB832702), the National Science Funds for Distinguished Young Scholars of China (11125208), the R\&D Special Fund for Public Welfare Industry (Hydrodynamics, Project No. 201101014), the 111 project under grant B12032, and Jiangsu Province Graduate Students Research and Innovation Plan (No. CXZZ11_0424).

\section{References}

[1] Luo J.F., Liu Y.J. and Berger E.J. Interfacial stress analysis for multicoating systems using an advanced boundary element method. Computational Mechanics, 24(6), pp. 448-455, 2000.

[2] Cheng A.H.D. and Cheng D.T. Heritage and early history of the boundary element method. Engineering Analysis with Boundary Elements, 29(3), pp. 268-302, 2005.

[3] Fairweather G. and Karageorghis A. The method of fundamental solutions for elliptic boundary value problems. Advances in Computational Mathematics, 9(1), pp. 69-95, 1998.

[4] Chen C.S., Golberg M.A. and Hon Y.C. The method of fundamental solutions and quasi-Monte-Carlo method for diffusion equations. International Journal for Numerical Methods in Engineering, 43(8), pp. 1421-1435, 1998. 
[5] Marin L., Karageorghis A. and Lesnic D. The MFS for numerical boundary identification in two-dimensional harmonic problems. Engineering Analysis with Boundary Elements, 35(3), pp. 342-354, 2011.

[6] Young D.L., Chen K.H. and Lee C.W. Novel meshless method for solving the potential problems with arbitrary domain. Journal of Computational Physics, 209(1), pp. 290-321, 2005.

[7] Chen W. and Wang F.Z. A method of fundamental solutions without fictitious boundary. Engineering Analysis with Boundary Elements, 34(5), pp. 530-532, 2010.

[8] Gu Y., Chen W. and He X.-Q. Singular boundary method for steady-state heat conduction in three dimensional general anisotropic media. International Journal of Heat and Mass Transfer, 55(17-18), pp. 48374848, 2012.

[9] $\mathrm{Gu} \mathrm{Y}$. and Chen W. Infinite domain potential problems by a new formulation of singular boundary method. Applied Mathematical Modelling, 37(4), pp. 1638-1651, 2013.

[10] Gu Y., Chen W. and Zhang C.-Z. Singular boundary method for solving plane strain elastostatic problems. International Journal of Solids and Structures, 48(18), pp. 2549-2556, 2011.

[11] Gu Y., Chen W. and Zhang J. Investigation on near-boundary solutions by singular boundary method. Engineering Analysis with Boundary Elements, 36(8), pp. 1173-1182, 2012.

[12] $\mathrm{Gu}$ Y. and Chen W. Infinite domain potential problems by a new formulation of singular boundary method. Applied Mathematical Modelling, 37(4), pp. 1638-1651, 2013.

[13] Zhang Y.-M., Gu Y. and Chen J.-T. Boundary element analysis of 2D thin walled structures with high-order geometry elements using transformation. Engineering Analysis with Boundary Elements, 35(3), pp. 581-586, 2011.

[14] Johnston B.M., Johnston P.R. and Elliott D. A sinh transformation for evaluating two-dimensional nearly singular boundary element integrals. International Journal for Numerical Methods in Engineering, 69(7), pp. 1460-1479, 2007.

[15] Luo J.F., Liu Y.J. and Berger E.J. Analysis of two-dimensional thin structures (from micro- to nano-scales) using the boundary element method. Computational Mechanics, 22(5), pp. 404-412, 1998.

[16] Sladek V., Sladek J. and Tanaka M. Nonsingular BEM formulations for thin-walled structures and elastostatic crack problems. Acta Mechanica, 99(1-4), pp. 173-190, 1993.

[17] Gu Y., Chen W. and Zhang C-Z. The sinh transformation for evaluating nearly singular boundary element integrals over high-order geometry elements. Engineering Analysis with Boundary Elements, 37(2), pp. 301308, 2013.

[18] Gu Y., Chen W. and Zhang C-Z. Stress analysis for thin multilayered coating systems using a sinh transformed boundary element method. International Journal of Solids and Structures, 50(20-21), pp. 3460-3471, 2013. 